\title{
Anti-lamin A/C antibodies in patients with Behçet's disease
}

\author{
PENG CHEN, HAI YAN, WEN ZHANG, CHUNHE YANG, HONGWU DU
}

School of Chemistry and Biotechnology Engineering, University of Science and Technology Beijing, Beijing, China

\begin{abstract}
Lamin A/C has been identified as a target antigen of anti-endothelial cell antibodies in vasculitis. Behçet's disease $(B D)$ is a chronic vasculitis with unclear pathogenesis. Self-antibodies have been found to play an important role in the pathological process of BD. The aim of this study was to verify whether lamin $A / C$ is an autoantigen of BD. First, the human lamin A/C protein was cloned and purified and verified by Western blotting. Next, a homemade ELISA kit was used to test the presence of anti-lamin $A / C$ antibodies in $B D$ patients. This is the first time it has been certified that lamin $A / C$ is an autoantigen of $B D$. The reactivity of serum IgG against recombinant human lamin $A / C$ was detected in 7 of $31 B D$ patients (23\%) and 3 of 32 healthy controls (HC) (9\%).
\end{abstract}

Key words: Behçet's disease, autoantigen, lamin A/C.

(Centr Eur J Immunol 2018; 43 (1): 358-361)

\section{Introduction}

Recently, lamin $\mathrm{A} / \mathrm{C}$ was identified as a target antigen of anti-endothelial cell antibodies (AECA) in patients with anti-neutrophil cytoplasmic antibody associated vasculitides [1]. In previous studies, anti-lamin $\mathrm{A} / \mathrm{C}$ antibodies were also detected in patients with pulmonary hypertension, scleroderma and giant cell arteritis [2]. These findings indicate that lamin $\mathrm{A} / \mathrm{C}$ might be widely present in autoimmune diseases as a monitoring molecule for the nuclear membrane.

Behçet's disease (BD) is a chronic vasculitis characterized by the triple-symptom complex of recurrent oral and genital ulcerations and ophthalmitis [3]. Self-antibodies which can activate the endothelial cells and the inflammatory response have been found to play an important role in the pathological process of BD [4]. Recently, four target antigens of AECA in BD - prohibitin, ETFB, HSP27 and annexin A2 - have been successfully identified in our laboratory [5-8]. However, more potential immune targets should be found to understand the exact pathogenesis of BD. Based on this, here we focused on two questions: whether lamin $\mathrm{A} / \mathrm{C}$ is an AECA autoantigen of $\mathrm{BD}$ and, if so, in what frequency.

\section{Material and methods}

\section{Subjects}

In this study, the subjects included $31 \mathrm{BD}$ patients with an average age of 39 years (range 19 to 61,16 female and 15 male), who all fulfilled the criteria proposed by the International Study Group for BD in 1990 [9], 24 systemic lupus erythematosus (SLE) patients (average 32 years old: range 16 to 54, 20 female and 4 male) and 32 healthy controls (average 26 years old: range 21 to 32, 28 female and 4 male). The BD patients and the controls were all collected in the Chinese PLA General Hospital before 2014. This study was approved by the ethical committee of the Chinese People's Liberation Army General Hospital; each patient involved gave informed consent. All the donated samples were dispensed and stored at $-80^{\circ} \mathrm{C}$ before further tests. Five BD patients and five healthy controls were randomly selected to verify whether lamin A/C is an autoantigen of BD by Western blotting.

\section{Protein cloning, expression and purification}

Total RNA was isolated from EA.hy926 cells using TRIzol reagent (Invitrogen, CA). RT-PCR was carried out according to the manufacturer's instructions (Fermentas, MD). Primer sequences were 5'-CCTGAATTCCCCAAAACGATCAGTGTG-3' for the sense primer and 5'-CCCAAGCTTTTATTAGCGCAGGGTCTTGTATTT-3' for the antisense. Human lamin A/C protein was overexpressed in Escherichia coli BL21, followed by the purification of recombinant proteins using Ni-NTA resin (Qiagen, Hilden, Germany). Purified recombinant protein was confirmed by AB4700 mass spectrometry (Applied Biosystems, Foster City, CA).

\section{Western blotting}

Western blotting was used as a preliminary experiment to test the value of the antigen. Recombinant human lamin A/C protein was loaded into the wells of a $12 \%$ polyacryl-

Correspondence: Prof. Hai Yan, School of Chemistry and Biotechnology Engineering, University of Science and Technology Beijing, 30 Xueyuan Road, Haidian District, Beijing 100083, China, e-mail: haiyan@ustb.edu.cn Submitted: 16.03.2016, Accepted: 9.04.2018 
amide gel and separated. The gel was then transferred onto polyvinylidene fluoride membranes (Merck Millipore, MA) that had been washed twice with ultrapure water. The membranes were then blocked with $5 \%$ nonfat milk in PBS at $4^{\circ} \mathrm{C}$ overnight and then incubated with $5 \mathrm{BD}$ sera (1 : 500 dilutions) or sera from $\mathrm{HC}$ at $4^{\circ} \mathrm{C}$ over $12 \mathrm{~h}$. The membranes were extensively washed 4 times with $5 \%$ PBST buffer to remove unbound antibodies. Last, they were incubated with horseradish-peroxidase-conjugated goat anti-human IgG (ImmunoHunt, Beijing, China) for $1 \mathrm{~h}$ at $37^{\circ} \mathrm{C}$, and ECL detection was carried out in accordance with the product instructions (Applygen, Beijing, China).

\section{ELISAs}

A homemade ELISA kit was optimized and evaluated to test the presence of anti-lamin $\mathrm{A} / \mathrm{C}$ antibodies in BD patients. Recombinant lamin A/C protein (100 ng/ml) was used to coat the 96-well microplates (Corning, NY) overnight at $4^{\circ} \mathrm{C}$. After three washes with PBST, each well was blocked in $200 \mu \mathrm{l} 5 \%$ goat serum for $2 \mathrm{~h}$ at $37^{\circ} \mathrm{C}$. Then the plate was incubated with $100 \mu$ sera diluted $1: 100$ in PBS for $2 \mathrm{~h}$ at $37^{\circ} \mathrm{C}$. Three washes later, $100 \mu \mathrm{l}$ of goat anti-human IgG/HRP (ImmunoHunt, Beijing, China) was added to each well and the plate was then incubated for an additional $1 \mathrm{~h}$ at $37^{\circ} \mathrm{C}$. The absorbance of each well was measured with a plate reader at $450 / 620 \mathrm{~nm}$ (Tecan, Hombrechtikon, Switzerland).

\section{Statistical analysis}

Fisher's exact test was performed using GraphPad Prism. $P$ values less than 0.05 were considered statistically significant. The cut-off value was obtained based on ROC analysis, which was carried out between BD patients and healthy controls with MedCalc software.

\section{Results}

As a result of Western blotting, an $\sim 70 \mathrm{kDa}$ band was observed in 2 of 5 BD patients, but no binding signal observed for $\mathrm{HC}$ (Fig. 1A). Reactivity of serum IgG against recombinant human lamin $\mathrm{A} / \mathrm{C}$ was detected in 7 of $31 \mathrm{BD}$ patients (23\%), 5 of 24 SLE patients $(21 \%)$, and 3 of 32 $\mathrm{HC}$ (Fig. 1B). The interaction network containing lamin $\mathrm{A} / \mathrm{C}$ and other BD autoantigens identified in our laboratory is shown (Fig. 1C).

\section{Discussion}

The exacted mechanism of lamin $\mathrm{A} / \mathrm{C}$ in the pathological process of diseases has not been fully revealed. Its important physiological functions may be the key to understand its pathological value. As the major components of the nuclear lamina, lamin $\mathrm{A} / \mathrm{C}$ is a fibrous layer on the nucleoplasm side of the inner nuclear membrane, which is thought to provide a framework for the nuclear envelope and may also interact with chromatin. It plays an important role in nuclear assembly, chromatin organization, nuclear membrane and telomere dynamics. Lamin $\mathrm{A} / \mathrm{C}$ has been identified as an AECA antigen in many autoimmune diseases, and now it is confirmed to be a new immune target for some patients with BD. The induction of lamin A/C autoantibodies is related to various symptoms [10].

We think the conclusion of this study is "two commons". First, lamin A/C might be a common antigen in many vasculitis diseases, without significant distribution characteristics $[2,11]$. Because of the nucleus location, it may play a common and non-innate role in the process of these diseases. Using the string pathway analysis database (http://string-db.org/), an interaction network containing lamin $\mathrm{A} / \mathrm{C}$ and other BD autoantigens identified in our laboratory was identified (Fig. 1C). From this network, we found that lamin $\mathrm{A} / \mathrm{C}$ may be triggered by other autoantigens as a secondary autoimmune response. $\beta$-actin, another common antigen in BD and other autoimmune diseases, was found to be a key intermediate molecule in this network. Second, BD may share some common pathological pathways with other vasculitides. So far, about twenty autoantigens in $\mathrm{BD}$ patients have been found. Many of them co-existed in many vasculitides such as ENO1, HNRNP A2/B1 [4], RLIP76 [12] and lamin A/C identified in this study. This further illustrated the vasculitis property of BD, and proved the key role of endothelial cells and AECA antigens in the process of BD development again.

\section{Conclusions}

Although the induced anti-lamin $\mathrm{A} / \mathrm{C}$ antibodies do not have statistical significance in patients' blood circulation, these findings confirm our original hypothesis, that lamin $\mathrm{A} / \mathrm{C}$ does play a role in BD. However, there are many weak points in this study. In 2014 the new international criteria for $\mathrm{BD}$, which have higher sensitivity than last edition, were published [13]. Because the samples used in this study were all collected before 2014, we checked the clinical data of samples in this study again. We found they all fulfilled the criteria set in 1990 and 2014. We cannot take new samples from those patients who fulfill the old criteria but not the new, and we do not know what the results may be in this category of BD patients. The high specificity of AECA autoantigen in BD still needs to be further explored with more sensitive bio-technologies.

\section{Acknowledgements}

This work was supported by the National Natural Science Foundation of China (No. 81571592, 31371203), Beijing Nova Program of Science and Technology (No. 2007B024), and the Program for New Century Excellent Talents in University (No. NECT-12-0773). 

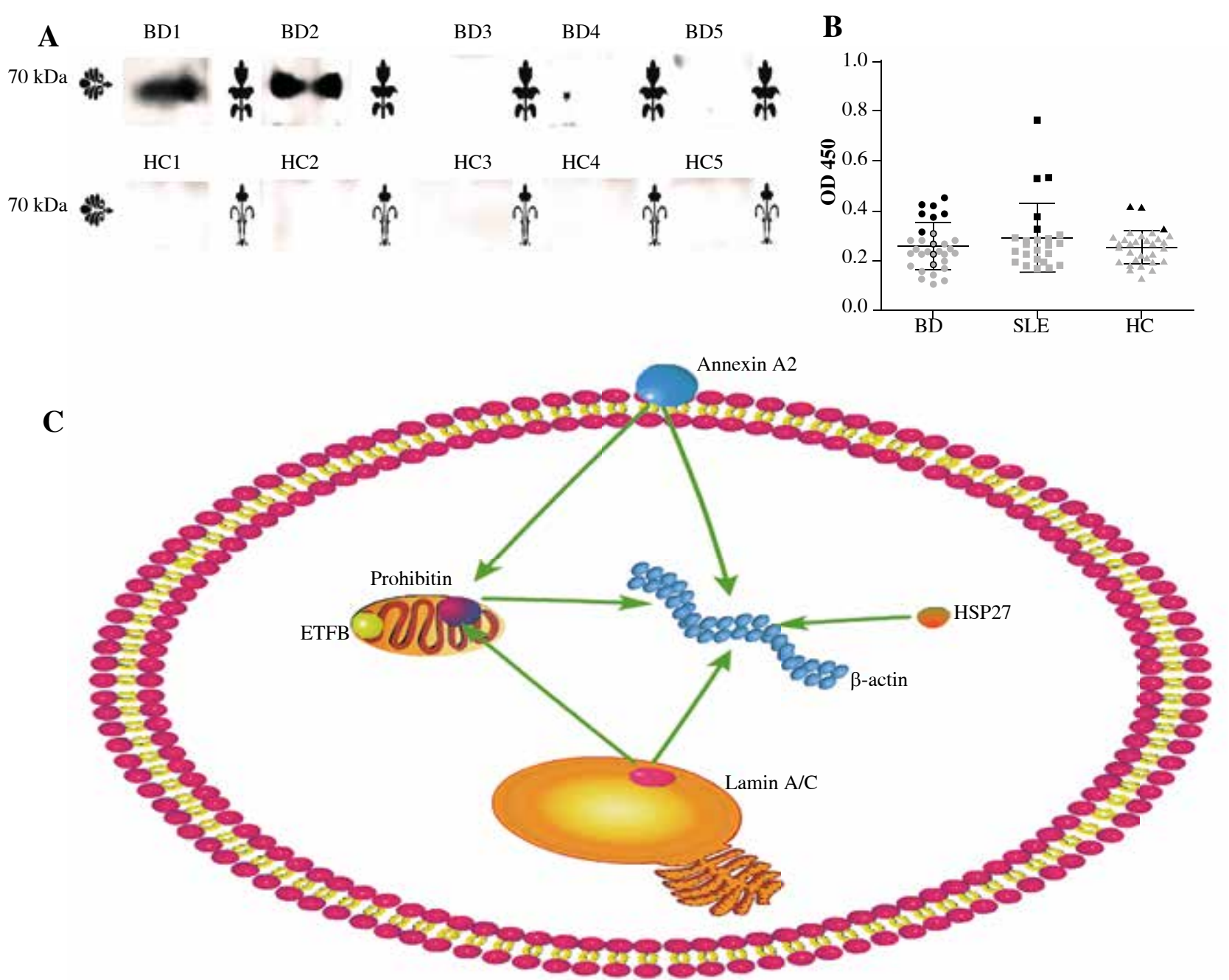

BD - Behçet's disease, HC - healthy controls,

SLE - systemic lupus erythematosus

Fig. 1. Lamin A/C was verified as an autoantigen of Behçet's disease. A) Western blotting of lamin A/C probed with patients' sera. B) Reactivity of sera IgG antibodies against recombinant human lamin A/C protein was detected by ELISA method. Data are expressed as mean \pm SD. C) Interaction network containing lamin A/C and other BD autoantigens identified in our laboratory is shown

The authors declare no conflict of interest.

\section{References}

1. Régent A, Lofek S, Dib H, et al. (2014): Identification of target antigens of anti-endothelial cell antibodies in patients with anti-neutrophil cytoplasmic antibody-associated vasculitides: A proteomic approach. Clin Immunol 153: 123-135.

2. Régent A, Dib H, Ly KH, et al. (2011): Identification of target antigens of anti-endothelial cell and anti-vascular smooth muscle cell antibodies in patients with giant cell arteritis: a proteomic approach. Arthritis Res Ther 13: R107.
3. Ayděntug AO, Tokgöz G, D'Cruz DP, et al. (1993): Antibodies to endothelial cells in patients with Behçet's disease. Clin Immunol Immunopathol 67: 157-162.

4. Cho SB, Ahn KJ, Kim do H, et al. (2012): Identification of HnRNP-A2/B1 as a target antigen of anti-endothelial cell $\mathrm{IgA}$ antibody in Behcet's disease. J Invest Dermatol 132: 601-608.

5. Chen P, Yang W, Tian Y, et al. (2015): Electron transfer flavoprotein subunit beta is a candidate endothelial cell autoantigen in Behçet's disease. PLoS One 10: e0124760.

6. Chen P, Shi L, Jiang Y, et al. (2014): Identification of heat shock protein 27 as a novel autoantigen of Behçet's disease. Biochem Biophys Res Commun 456: 866-871. 
7. Chen P, Yan H, Tian Y, et al. (2015): Annexin A2 as a target endothelial cell membrane autoantigen in Behçet's disease. Sci Rep 5: 8162.

8. Xun Y, Chen P, Yan H, et al. (2014): Identification of prohibitin as an antigen in Behcet'sdisease. Biochem Biophys Res Commun 451: 389-393.

9. International Study Group for Behçet's Disease (1990): Criteria for diagnosis of Behçet's disease. Lancet 335: 1078-1080.

10. Goizet C, Yaou RB, Demay L, et al. (2004): A new mutation of the lamin $\mathrm{A} / \mathrm{C}$ gene leading to autosomal dominant axonal neuropathy, muscular dystrophy, cardiac disease, and leuconychia. J Med Genet 41: e29.

11. Nesher G, Margalit R, Ashkenazi YJ (2001): Anti-nuclear envelope antibodies: clinical associations. Semin Arthritis Rheum 30: 313-320.

12. Goizet C, Yaou RB, Demay L, et al. (2008): Autoantibodies to the C-terminal subunit of RLIP76 induce oxidative stress and endothelial cell apoptosis in immune-mediated vascular diseases and atherosclerosis. Blood 111: 4559-4570.

13. International Team for the Revision of the International Criteria for Behçet's Disease (ITR-ICBD), (2014): The International Criteria for Behçet's Disease (ICBD): a collaborative study of 27 countries on the sensitivity and specificity of the new criteria. J Eur Acad Dermatol Venereol 28: 338-347. 